\title{
Endogenous vascular repair system in cardiovascular disease: The role of endothelial progenitor cells
}

Alexander E Berezin

Internal Medicine Department, State Medical University, Zaporozhye, Ukraine

\section{REVIEW}

Please cite this paper as: Berezin AE. Endogenous vascular repair system in cardiovascular disease: The role of endothelial progenitor cells. AMJ 2019;12(2):42-48.

https://doi.org/10.21767/AMJ.2018.3464

\section{Corresponding Author:}

Alexander E Berezin

Internal Medicine Department, State Medical University, Zaporozhye, 69035, Ukraine

Email: aeberezin@gmail.com

\section{ABSTRACT}

\section{Background}

Cardiovascular (CV) disease has been considered as the first global leading cause of premature death in last decades. Vascular complications close correspond to development of endothelial dysfunction, microvascular inflammation, accelerating atherosclerosis, coagulopathy, and thrombosis, in pathogenesis of which altered vascular reparation is core player and possible target for further treatment strategy.

\section{Aims}

The aim of the review is: to summarize knowledge regarding the role of endothelial progenitor cells (EPCs) as a core component of endogenous vascular repair system in CV disease.

\section{Methods}

This is a descriptive review based on evidence of pre-clinical and clinical studies published within last decade.

\section{Results}

The review is reported that developing of CV disease associates with lowered number and impaired function of circulating EPCs, which play a pivotal role in endogenous reparation of vasculature and restore endothelial function after various injuries on the vessels. EPCs could be modified by several stimuli including epigenetic factors and thereby they are failed completely restoring vascular structure and endothelial function. However, there is unclear whether EPC dysfunction is just a whiteness of the evolution of $\mathrm{CV}$ disease or it could be a trigger of CV disease manifestation in the vulnerable population.

\section{Conclusion}

EPCs are novel biological marker of impaired vascular reparation and CV risk with predictive value. Large clinical trials are required to justify number and function of EPCS as independent prognosticator of CV risk.

\section{Key Words}

Cardiovascular disease, endothelial dysfunction, vascular remodelling, vascular reparation, endothelial progenitor cells

\section{What this review adds:}

\section{What is known about this subject?}

Endothelial progenitor cells are involved in vascular reparation and angiogenesis. Development of cardiovascular disease associates strongly with lowered number and weak function of circulating precursors.

\section{What new information is offered in this review?}

This review explores progenitor endothelial cell dysfunction as a cause of altered vascular reparation in cardiovascular disease, and suggests how to improve it.

3. What are the implications for research, policy, or practice?

Endothelial progenitor cells may be diagnostic and predictive biological markers of cardiovascular outcomes, and potent target for the treatment.

\section{Introduction}

Cardiovascular (CV) disease remains a leading cause of premature death and disability in numerous countries and 
regions worldwide. ${ }^{1}$ Although $\mathrm{CV}$ mortality rates has been demonstrated graduate declining for last decade, increase of both life span and populations' age predominantly in developed countries associates with rising non-fatal CV clinical outcomes and their consequences including disability and all these are becoming a core component of the global and regional burden of CV disease. ${ }^{2}$ Vascular complications strong relate to endothelial dysfunction, microvascular inflammation, accelerating atherosclerosis, coagulopathy, and thrombosis. All these pathophysiological conditions are several faces of universal process called vascular remodelling that corresponds to nature evolution of CV disease and it is a result of the imbalance between vascular injury and vascular reparation. In this context, residential or circulating precursors with high proliferative capability and angiopoetic potency are considered key players in restoring of vascular structure and function. The aim of the review is: to summarize knowledge regarding the role of endothelial progenitor cells as a core component of endogenous vascular repair system in CV disease.

\section{Definition of vascular remodelling}

Vascular remodelling plays a pivotal role in CV disease affecting several phases (genetic/epigenetic, sub-molecular, molecular, cellular, and histomophometric) of the pathogenesis of one. ${ }^{3}$ At early stages of CV disease evolution the altered vascular function appears as endothelial dysfunction, which was recognised an independent CV risk factor with important predictive value regardless of ethiology of CV disease. Progression of endothelial dysfunction is strongly associated with thickening of the vascular wall due to proliferative changes in the intima and media, infiltration of lipids, accumulation of pro-inflammatory cytokines, chemokines and macrophages (mononuclears), extracellular matrix deposition of collagen and occurrence of fibrosis., ${ }^{3,4}$ Later, modifications of normal anatomical architectonic and micro aneurism can occur, loss of capillary greed with increasing inter-capillary dimensions, lowering perfusion efficacy and inducing tissue ischemia/ hypoxia. Therefore, micro neovascularization as a result of hypoxia, accumulation of activated mononuclears in intima and sub-intima, triggers plaque instability, haemorrhage and eventually atherothrombosis. ${ }^{5}$ Finally, rigid vascular wall with altered vasodilation including capillary rarefaction and increased aortic diameter are usually described as core components of vascular remodelling accompanying with nature evolution of the CV disease. ${ }^{6}$

Vascular remodelling is triggered by wide spectrum of stimuli, such as mechanical (high blood pressure, shear stress), metabolic (lipids, hyperglycaemia, hyperuricemia, hyper-and hypothyroidisms, hyperphosphatemia), inflammatory (reactive oxygen species, inflammatory cytokines and chemokines), ischemia / hypoxia that may also close associate with senescence and vascular aging. ${ }^{7-9}$

There is large body of evidence of triggers able to epigenetically modify precursors, a process by which cells stop proliferating and become dysfunctional. ${ }^{10}$ In this context, vascular reparation is "impaired" and associated with dysfunctional responses, such endothelial dysfunction.

\section{Conception of endogenous vascular repair system}

Vascular reparation is an attribute of plasticity of bonemarrow and/ or peripheral tissue residence/ progenitor cells including precursors of vascular smooth muscle cells endothelial cell. ${ }^{11}$ The growth, mobbing, proliferation, (trans)-differentiation, apoptosis and survival of cell precursors are under tight auto-paracrine control, remarkable epigenetic regulation (microRNAs, DNA/ histone methylation), supporting by direct cell-to-cell cooperation and micro-vesicle-related cargo transfer. ${ }^{12}$ Subsequently, vascular reparation is combined pathophysiological process based on correspondence to cells, sub-cellular elements, and circulating auto-paracrine factors. The core of vascular repair system belongs to endothelial progenitor cells (EPCs), which are actively investigated last two decades and continually pay attention of scientists as possible biological markers of vascular reparation, endothelial dysfunction, angiogenesis/ neovascularization, vascular complications with likely predictive value.

\section{Progenitor endothelial cells}

Fist pioneer investigations that confirmed being of special populations of the circulating cells with impressive angiogenic abilities were provided by Asahara et al. ${ }^{13}$ Using animal models of ischemia authors found sites of active angiogenesis with incorporated into its putative EPCs or angioblasts, which were further isolated from peripheral blood of animals and humans by magnetic bead selection on the basis of cell surface antigen expression. ${ }^{14,15}$ These cells have demonstrated an high ability to differentiation onto an endothelial lineage in colonies in culture, and in animal studies these cells have been incorporated into active cores of neovascularization demonstrating impressive capability to attenuate angiogenesis and vascular function in vivo through differentiation into mature endothelial cells. ${ }^{15,16}$ Taking into consideration the origin of these cells from bone marrow stem cells and human umbilical cord blood and that fact they may mobilize and migrate from bone marrow and differentiate into mature endothelial cells the population of these precursors were called EPCs. Later it 
has known that EPCs may transdifferentiate into cells with phenotype distinguished from mature endothelial cells, for example, smooth muscle cells of the media of vessels. ${ }^{17}$ Therefore, EPCs are able to synthase and realize a wide range of active molecules (E-selectin, P-selectin), peptides and growth factors (vascular endothelial growth factor, fibroblast growth factor, granulocyte-macrophage colonystimulating factor) that modulate angiogenesis and improve vascular integrity and function. ${ }^{17,18}$ Thus, the main biological role of EPCs is improving of vascular reparation and angiogenesis.

\section{Populations of endothelial progenitor cells}

To verify different populations of EPCs early studies were used several techniques based on determination of appropriate molecular features, i.e. expression of antigens. Before appearance of consensus statement regarding common strong endothelial markers, which are expressed on the surface of these precursors, there was not agreement about immune phenotypes of EPCs. ${ }^{19}$ It has been postulated that EPCs must not express CD45 antigen (hematopoietic marker), but obligatory have other markers of endothelial cells, such as CD31 (platelet/endothelial cell adhesion molecule-1), CD144 (vascular cadherin), CD309 (vascular endothelial growth factor receptor-2), and CD133 (prominin-like 1). ${ }^{20,21}$ Later it has established that CD45(-) cells simultaneously co-expressing CD34 (MY10), CD133 and CD309, referred to as EPCs. ${ }^{22}$ Although CD133, CD34, and CD309 are referred constitutive markers for EPCs, some populations of EPCs may lose CD133 antigen within differentiation period and may express on their surface other antigens, i.e. CD31, CD144, endothelial NO synthase (eNOs) and von Willebrand factor (vWf). Therefore, some fractions of EPCs with high proliferating capacity could contain non-hematopoietic population of CD45(-)CD31(+) cells with additional presentation CD117(+). Taken together, combination of CD45(-), CD31(+), CD 133(+), CD34(+) and CD309(+) or $\operatorname{vWf}(+)$ probably allows getting reliable purification of progenitor endothelial subsets from mononuclear cells with presentation of endothelial markers. ${ }^{21-23}$ The molecular features of EPCs are reported in detail in Table 1. Thus, different populations of EPCs may sufficiently distinguish each other not just depending on their origin, but according to stage of differentiation into mature endothelial cells or smooth muscle cells.

Additionally, EPCs expressed sufficient distinguish in selfrenewal ability that is determining by colony-forming technique. ${ }^{23}$ According to ability to appear in fibronectin coated dish all EPCs were divided into early outgrowth EPCs (5-7 days after fibronectin plating) or late outgrowth EPCs
(7-10 days after fibronectin plating). Interestingly, the late outgrowth EPCs originated from peripheral blood mononuclears demonstrated CD31(+)CD146(+)CD105(+) and/ or $\mathrm{CD} 309(+)$ immune phenotype and had functional properties suitable for mature endothelial cells. Depending on presentation on the cell surface CD34 antigen all late outgrowth EPCs are divided into two populations. The population of EPCS with immune phenotypes CD34(+)CD31(+), CD34(+)CD31(+)CD146(+), CD34(+)CD31(+), CD105(+), and CD34(+)CD31(+)CD309(+) demonstrated higher proliferative potency to CD34(-) EPCs co-expressing CD31, CD309, CD105 and CD146 antigenes. Therefore, CD34(+) EPCs had reproduced tubes and colony shaping in the single-cell colony-formation investigation as well as they responded to angiogenic growth factors. ${ }^{24}$ In contrast, CD34(-) cells had limited ability to reproduce colonies or even had not these properties in vitro. There is evidence regarding that CD34(-) EPCs in the colony lead to cultures collapsed within one or two passages that confirm an idea of strong hierarchy in self-renewal EPCs may be an extremely important functional feature of endothelial cells' precursors. $^{25}$ Finally, the population of EPCs, which originates from the bone marrow hemangioblasts are released into peripheral blood and involved in vascular repair. Other candidates for cell surface molecule markers and genes' signatures to a functional determination based surely on self-renewal hierarchy of EPCS are actively investigated. ${ }^{26}$ Thus, there are several populations of EPCS with different proliferative activities and angiopoetic potencies that can be seized upon biological markers of vascular reparation, vessel injury and endothelial dysfunction.

\section{Molecular mechanisms of vascular reparation: focus on endothelial progenitor cells}

The mobilization, differentiation, proliferation, adhesion, survival / apoptosis and incorporation into endothelial cell monolayers of EPCs are enhanced by numerous factors (vascular endothelial growth factor [VEGF], hypoxiainducible factor-alpha [HIF-alpha], stromal cell-derived factor-1 [SDF-1], transforming growth factor beta-1 [TGFbeta1], metalloproteinases [MMP] and their tissue inhibitors, inflammatory cytokines [interleukine-1 beta, tumour necrosis factor-alpha, etc.]) that act predominantly through p57 VEGF/eNOs and HIF/p-Akt/p-eNOS/MMP-9 signalling system and Janus kinase-2/ CXCR4 axis and chemokine SDF-1 Akt-related pathways. ${ }^{27}$ In fact, increased expression of cdkn1c and il33 genes of Notch signalling play a core role in regulation of cell cycling with EPCs and bone marrow hemangioblasts. ${ }^{28}$ Additionally, circulating EPCS may directly contact with the injury sites of endothelium 
and trigger local proliferation of residence cells, promote reendothelization and angiogenesis. ${ }^{29}$ These effects are mediated by secretome of EPCs, which includes micro vesicles with regulatory proteins, peptides, micro-RNAs, growth factors (VEGF, HIF-alpha, TGF-beta1), hormones (aldosterone, endothelin), and wide spectrum of active molecules (E-selectin, P-selectin). ${ }^{30}$ Indeed, EPCs differentiate into mature endothelial cells and immediately become a target for inflammatory cytokines, factors of coagulation, hormones (catecholamines, aldosterone, angiotensin-II, endothelin), active molecules that have ability to induce apoptosis. Apoptotic endothelial cells switch over secretion of micro vesicles to nanoparticles containing chromatin, methylated RNAs and DNAs. Apoptotic-related nanoparticles produced by mature endothelial cells directly injury an endothelium and mediate inflammation and coagulation cascades. In contrast, but micro vesicles that are released by activated mature endothelial cells enhance a vascular reparation and attenuate endothelial function. ${ }^{31}$ Overall, EPCs with angiopoetic phenotypes may contribute with vasculature with direct and indirect ways depending on pre-existing ability of EPCs to proliferation, differentiation and survival as well as spectrum of co-regulatory factors, which mainly alter maturation and commitment of stem cells/ progenitor precursors and impair EPC mobilization.

\section{Endothelial progenitor cell dysfunction as novel marker of cardiovascular risk}

Decreased number, lowered survival and/ or weak function of circulating EPC was described as EPC dysfunction. ${ }^{32}$ This phenomenon associated with altered vascular structure/ function and related to senescence, asymptomatic atherosclerosis, CV disease, chronic kidney disease, metabolic syndrome, diabetes mellitus, and hyperthyroidism. $^{33,34}$ Although EPC dysfunction accompanies a number of CV risk factors, whether altered vascular reparation appears to be prior to $\mathrm{CV}$ disease or it is a result of influence of $\mathrm{CV}$ risk factors is not fully clear. It has been postulated that the EPC dysfunction is not just attribute of time period, in which EPCs has been contacting with CV risk factors, but it could be a pre-emptive adaptation to epigenetic stimuli corresponding to vascular remodelling. As a result, the repair ability of vasculature is dramatically lowered due to failed ability of EPCs differentiate to mature endothelial cells and completely restore vascular integrity and function. Finally, endothelial dysfunction persists and damage target organs leading to increased CV risk.

Indeed, there is large body of evidence regarding the fact that EPC dysfunction is additional CV risk factor with ability to predict CV mortality rate, a risk of urgent hospital admission and newly diagnosed CV events. ${ }^{35}$ Although majority of $\mathrm{CV}$ and metabolic diseases associated with lowered number of circulating EPCs, there are conditions (acute heart failure, acute myocardial infarction, acute pulmonary thromboembolism, type 1 diabetes mellitus) related to unpredictable changes of number of EPCs. ${ }^{36,37}$

It has suggested that the number of urgently recruited bone-marrow precursors can be limited due to previous expenditures for vascular reparations, which were prior to CV events, i.e. diabetes, traumas and infections. ${ }^{38}$ Additionally, ischemia/ hypoxia, several spectrum of proinflammatory cytokines and growth factors increase both mobbing of precursors from bone marrow and their proliferative capability. ${ }^{39,40}$ Final results may relate to pole of precursors and severity of epigenetic modification of them. In this context, it is extremely important avoid calculating number of circulating EPCs and implementing assay of their function. However, EPC dysfunction is novel biological marker of endothelial dysfunction and vascular reparation in CVD with predictive value requires to be investigated in large clinical trials.

\section{Conclusion}

Development and progression of CV disease are associated with altered vascular structure and function that consequently correspond to vascular complications and risk of unfavourable clinical outcomes. Endothelial progenitor cells are involved in the vascular reparation and restoring vascular integrity and function as core component of endogenous repair system. Complicated co-regulation in mobbing, proliferation, differentiation, transformation of immature progenitor precursors into mature endothelial cells could be impaired by CV risk factors, epigenetic stimuli and co-morbidities, such as diabetes mellitus, metabolic syndrome, that mediate impaired survival, number and function of endothelial progenitor cells. Endothelial precursor dysfunction appears as novel CV risk factor with likely predictive value.

\section{References}

1. GBD 2016 Disease and Injury Incidence and Prevalence Collaborators. Global, regional, and national incidence, prevalence, and years lived with disability for 328 diseases and injuries for 195 countries, 1990-2016: a systematic analysis for the Global Burden of Disease Study 2016. Lancet. 2017;390(10100):1211-1259.

2. Roth GA, Johnson C, Abajobir A, et al. Global, regional, and national burden of cardiovascular diseases for 10 
causes, 1990 to 2015. J Am Coll Cardiol. 2017;70(1):125.

3. Sedding DG, Boyle EC, Demandt JAF, et al. Vasa vasorum angiogenesis: Key player in the initiation and progression of atherosclerosis and potential target for the treatment of cardiovascular disease. Front Immunol. 2018;9:706.

4. Dzau VJ, Braun-Dullaeus RC, Sedding DG. Vascular proliferation and atherosclerosis: new perspectives and therapeutic strategies. Nat Med. 2002;8(11):1249-56.

5. Libby P, Hansson GK. Inflammation and immunity in diseases of the arterial tree: players and layers. Circ Res. 2015;116(2):307-11.

6. Bloksgaard M, Thorsted B, Brewer JR, et al. Assessing collagen and elastin pressure-dependent microarchitectures in live, human resistance arteries by label-free fluorescence microscopy. J Vis Exp. 2018;(134). doi: 10.3791/57451.

7. Liu J, Kang $H, M a ~ X$, et al. Vascular cell glycocalyxmediated vascular remodeling induced by hemodynamic environmental alteration. Hypertension. 2018. doi: 10.1161/HYPERTENSIONAHA.117.10678.

8. Salazar G. NADPH oxidases and mitochondria in vascular senescence. Int J Mol Sci. 2018;19(5). doi: 10.3390/ijms19051327.

9. Wu TW, Liu CC, Hung CL, et al. Genetic profiling of young and aged endothelial progenitor cells in hypoxia. PLoS One. 2018;13(4):e0196572.

10. Lehners $M$, Dobrowinski $H$, Feil $S$, et al. cGMP signalling and vascular smooth muscle cell plasticity. J Cardiovasc Dev Dis. 2018;5(2). doi: 10.3390/jcdd5020020.

11. Berezin AE. Endothelial progenitor cells dysfunction and impaired tissue reparation: The missed link in diabetes mellitus development. Diabetes Metab Syndr. 2017;11(3):215-220.

12. Berezin AE. Preconditioned endothelial progenitor cells as biomarker of vascular reparation? Insights in Biomed. 2017; 2(1):4-7.

13. Asahara T, Murohara T, Sullivan A, et al. Isolation of putative progenitor endothelial cells for angiogenesis. Science. 1997;275:964-966.

14. Asahara T. Endothelial progenitor cells for vascular medicine. Yakugaku Zasshi. 2007;127(5):841-5.

15. Patel J, Donovan P, Khosrotehrani K. Concise review: Functional definition of endothelial progenitor cells: A molecular perspective. Stem Cells Transl Med. 2016;5(10):1302-1306.

16. Yi C, Xia W, Zheng Y, et al. Transplantation of endothelial progenitor cells transferred by vascular endothelial growth factor gene for vascular regeneration of ischemic flaps. J Surg Res. 2006;135(1):100-6.

17. Asahara T, Masuda H, Takahashi T, et al. Bone marrow origin of endothelial progenitor cells responsible for postnatal vasculogenesis in physiological and pathological neovascularization. Circ Res. 1999;85(3):221-8.

18. Murohara T. Angiogenesis and vasculogenesis for therapeutic neovascularization. Nagoya J Med Sci. 2003;66(1-2):1-7.

19. Masuda H, Alev C, Akimaru $H$, et al. Methodological development of a clonogenic assay to determine endothelial progenitor cell potential. Circ Res. 2011;109(1):20-37.

20. Haberzettl P, Conklin DJ, O'Toole TE. Endothelial progenitor cells: properties, function, and response to toxicological stimuli. Comprehensive Toxicology (Third Edition) 2018;12:130-182. doi.org/10.1016/B978-0-12801238-3.64248-4.

21. Boldicke T, Tesar M, Griesel C, et al. Anti-VEGFR-2 scFvs for cell isolation. Single-chain antibodies recognizing the human vascular endothelial growth factor receptor-2 (VEGFR-2/flk-1) on the surface of primary endothelial cells and preselected CD34+ cells from cord blood. Stem Cells. 2001;19(1):24-36.

22. Ferreras C, Cole CL, Urban K, et al. Segregation of late outgrowth endothelial cells into functional endothelial CD34- and progenitor-like CD34+ cell populations. Angiogenesis. 2015;18(1):47-68.

23. Popa ER, Harmsen MC, Tio RA, et al. Circulating CD34+ progenitor cells modulate host angiogenesis and inflammation in vivo. J Mol Cell Cardiol. 2006;41(1):8696.

24. Xu QB. Endothelial progenitor cells in angiogenesis. Sheng Li Xue Bao. 2005;57(1):1-6.

25. Tagawa S, Nakanishi C, Mori M, et al. Determination of early and late endothelial progenitor cells in peripheral circulation and their clinical association with coronary artery disease. Int J Vasc Med. 2015;2015:674213.

26. Stroncek JD, Grant BS, Brown MA et al. Comparison of endothelial cell phenotypic markers of late-outgrowth endothelial progenitor cells isolated from patients with coronary artery disease and healthy volunteers. Tissue Eng Part A. 2009;15(11):3473-3486.

27. Hristov M, Erl W, Weber PC. Endothelial progenitor cells: mobilization, differentiation, and homing. Arterioscler Thromb Vasc Biol. 2003;23(7):1185-9.

28. Pasut A, Chang NC, Gurriaran-Rodriguez $U$, et al. Notch Signaling Rescues Loss of Satellite Cells Lacking Pax7 and Promotes Brown Adipogenic Differentiation. Cell Rep. 2016;16(2):333-343.

29. Fang S, Wei J, Pentinmikko N, et al. Generation of functional blood vessels from a single c-kit+ adult 
vascular endothelial stem cell. PLoS Biol. 2012;10:e1001407.

30. Qu K, Wang Z, Lin XL, et al. MicroRNAs: Key regulators of endothelial progenitor cell functions. Clin Chim Acta. 2015;448:65-73.

31. Berezin A. Epigenetics in heart failure phenotypes. BBA Clin. 2016;6:31-7.

32. Berezin $A E$, Kremzer $A A$, Berezina $T A$, et al. The pattern of circulating microparticles in patients with diabetes mellitus with asymptomatic atherosclerosis. Acta Clinica Belgica. 2016;71(1):38-45.

33. Boos CJ, Lip GYH, Blann AD. Circulating endothelial cells in cardiovascular disease. J Am Coll Cardiol. 2006;48:1538-47.

34. Dignat-George F, Sampol J. Circulating endothelial cells in vascular disorders: new insights into an old concept. Eur J Haematol. 2000;65:215-20.

35. Berezin AE, Kremzer AA. Circulating endothelial progenitor cells as markers for severity of ischemic chronic heart failure. J Card Fail. 2014;20(6):438-47.

36. Chong AY, Lip GYH, Freestone B, et al. Increased circulating endothelial cells in acute heart failure: comparison with von Willebrand factor and soluble Eselectin. Eur J Heart Fail. 2006;8:167-72.

37. Fadini GP, Sartore S, Baesso I, et al. Endothelial progenitor cells and the diabetic paradox. Diabetes Care. 2006;29:714-6.

38. Ling $L$, Shen $Y$, Wang $K$, et al. Worse clinical outcomes in acute myocardial infarction patients with type 2 diabetes mellitus: relevance to impaired endothelial progenitor cells mobilization. PLoS One. 2012;7(11):e50739.

39. Grochot-Przeczek A, Kotlinowski J, Kozakowska M, et al. Heme oxygenase-1 is required for angiogenic function of bone marrow-derived progenitor cells: role in therapeutic revascularization. Antioxid Redox Signal. 2014;20:1677-92.

40. Hoenig MR, Bianchi C, Sellke FW. Hypoxia inducible factor-1 alpha, endothelial progenitor cells, monocytes, cardiovascular risk, wound healing, cobalt and hydralazine: a unifying hypothesis. Curr Drug Targets. 2008;9(5):422-35.

\section{ACKNOWLEDGEMENTS}

None

\section{PEER REVIEW}

Not commissioned. Externally peer reviewed.

\section{FUNDING}

This research received no specific grant from any funding agency in the public, commercial, or not-for-profit sectors.

\section{ETHICS COMMITTEE APPROVAL}

Not applicable

\section{CONFLICTS OF INTEREST}

The author declares no competing interests. 
Table 1: Molecular makers for different populations of EPCs

\begin{tabular}{|l|l|l|l|}
\hline Antigen name & Alternative name & Distribution & Biological function \\
\hline CD31 & Endocam, PECAM-1 & $\begin{array}{l}\text { Monocytes, platelets, granulocytes, } \\
\text { lymphocytes subsets }\end{array}$ & $\begin{array}{l}\text { Cell adhesion, signal } \\
\text { transduction, receptor for CD38 }\end{array}$ \\
\hline CD117 & C-kit, SCFR & $\begin{array}{l}\text { Hematopoetic progenitor cells, mast } \\
\text { cells }\end{array}$ & $\begin{array}{l}\text { SCF receptor, regulator of } \\
\text { differentiation / proliferation of } \\
\text { hematopoetic progenitor cells }\end{array}$ \\
\hline CD34 & MY10, mucosialin & $\begin{array}{l}\text { Endothelial cells, embryonic } \\
\text { fibroblasts, hemapoetic precursors }\end{array}$ & $\begin{array}{l}\text { Stem cell marker, adhesion, } \\
\text { receptor for CD62 ligand }\end{array}$ \\
\hline CD105 & Endoglin & $\begin{array}{l}\text { Endothelial cells, bone marrow } \\
\text { subsets, activated macrophages }\end{array}$ & $\begin{array}{l}\text { Cellular response to TGF-beta1, } \\
\text { adhesion, embryonic } \\
\text { angiogenesis }\end{array}$ \\
\hline CD133 & AC133, prominin-like 1 & $\begin{array}{l}\text { Hemapoetic stem cell subsets, } \\
\text { endothelial cells, epithelial cells }\end{array}$ & Unknown \\
\hline CD144 & $\begin{array}{l}\text { VE-cadherin, cadherin- } \\
\text { E }\end{array}$ & Endothelial cells, stem cells & $\begin{array}{l}\text { Adhesion, endothelium integrity } \\
\text { and biology }\end{array}$ \\
\hline CD146 & MUC18, S-endo & Endothelial cells, melanomas & $\begin{array}{l}\text { Adhesion, target of CD44 } \\
\text { signalling }\end{array}$ \\
\hline CD309 & - & $\begin{array}{l}\text { Endothelial cells, angiogenic } \\
\text { precursors, hemangioblasts }\end{array}$ & $\begin{array}{l}\text { Binding VEGF, regulation of cell } \\
\text { adhesion and signalling, }\end{array}$ \\
\hline vWf & Endothelial cells & $\begin{array}{l}\text { Antihemophilic factor, platelet- } \\
\text { vessel wall mediator of } \\
\text { coagulation }\end{array}$ \\
\hline
\end{tabular}

Abbreviations: EPCs, endothelial progenitor cells; CD, cluster of differentiation; VEGFR, vascular endothelial growth factor receptor; MUC, melanoma cellular adhesion molecule; vWf, von Willebrand Factor; SCFR, Mast/Stem Cell Growth Factor Receptor; PECAM, platelet/endothelial cell adhesion molecule; TGF-beta1, transforming growth factor beta 1. 